\title{
Peculiarities of nanocrystalline silicon films growth on porous anodic alumina surface
}

\author{
P.V. Parfenyuk ${ }^{1}$, A.A. Evtukh ${ }^{1,2}$, I.M. Korobchuk ${ }^{2}$, V.I. Glotov ${ }^{3}$, V.V. Strelchuk ${ }^{1}$ \\ ${ }^{I}$ V. Lashkaryov Institute of Semiconductor Physics, NAS of Ukraine, Kyiv, Ukraine, \\ E-mail: anatoliy.evtukh@gmail.com \\ ${ }^{2}$ Institute of High Technologies, Taras Shevchenko National University of Kyiv, Kyiv, Ukraine \\ ${ }^{3}$ Institute of Microdevices, Kyiv, Ukraine
}

\begin{abstract}
The influence of porous alumina template morphology on silicon films growth at deposition by PE CVD has been investigated. As it was shown, the structural properties of silicon phases depend on the pore geometry and surface morphology of the anodized porous alumina substrate. In case of porous alumina formation in one stage, ripple-like morphology takes place. The growth of a-Si:H film is observed at deposition. After two-stage anodization, the porous alumina has tipped/ribbed morphology. In this case, usually a-Si:H film grows on the bottom of the pores, and nc-Si:H/a-Si:H one grows on the tips. In the case of deep pores, the nanocrystalline nc-Si:H film grows only above the top of the pores. The obtained results could be used when developing new types of photocells, sensors, nanophotonics and ionics devices.
\end{abstract}

Keywords: amorphous phase, nanocrystalline silicon films, porous anodic alumina, chemical vapor deposition, textured surface, Raman spectra.

Manuscript received 25.03.17; revised version received 07.08.17; accepted for publication 06.09.17; published online 09.10.17.

\section{Introduction}

The nanocrystalline silicon films are very attractive for optoelectronics, especially photovoltaics applications. As it was shown [1], the films of amorphous hydrogenized silicon that include the silicon nanocrystalls with the size of several nanometers and bulk concentration of several percents have higher sensitivity and stability in comparison with homogenous a-Si:H films. Significant advantages have been made in recent years to improve the efficiency of single and multi-junction solar cells incorporating nanocrystalline silicon (nc-Si:H). Nanocrystalline silicon is an adsorbing material, which is crucial for obtaining thin film tandem solar cells with a high efficiency $[2,3]$.

As a rule, nc-Si:H is obtained using the chemical vapor deposition (CVD) method. The variety of direct
CVD techniques has been used to yields materials with good optoelectronic properties [4-9]. But among them only plasma enhanced chemical vapor deposition (PE CVD) has been established for industrial applications [10]. The high RF-power and high hydrogen dilution are two critical parameters in conventional PE CVD method that facilitate nanocrystalization [11]. The high RFpower causes surface damage by high-energy ion bombardment, and high hydrogen dilution of silane retards the deposition rate of nc-Si:H films [12] and have constrained the film deposition to a narrow substrate temperature range [13]. The lower deposition rate increases the process operation time, and hence the cost whereas narrow substrate temperature range involves difficulty in controlling the hydrogen distribution in the film, which is responsible for light-induced degradation of electronic properties [14]. The new approach to form 
nc-Si:H films by PE CVD is based on the growth on textured substrates, namely porous anodic aluminum (PAA) substrates $[15,16]$.

The aim of this article is to study the surface morphology influence of the porous anodic alumina (PAA) substrate on the structural characteristics of the nanocrystalline silicon.

\section{Experimental}

\subsection{Preparation of porous anodic alumina templates}

To prepare the template of porous alumina substrate, the electrochemical oxidation of $0.05 \mathrm{~mm}$ thick aluminum foil was used. Before anodizing, the foil was degreased in acetone in ultrasonic bath, and then washed in deionized (DI) water.

The two-step anodizing processing was performed using $4 \% \mathrm{H}_{3} \mathrm{PO}_{4}$ electrolyte. In a first step of anodizing, Al foil samples (area $2 \times 2 \mathrm{~cm}^{2}$ ) were prepared in $4 \%$ $\mathrm{H}_{3} \mathrm{PO}_{4}$ under the following conditions: solution temperature $15^{\circ} \mathrm{C}$, anodizing voltage $130 \mathrm{~V}$, anodizing current $8 \mathrm{~mA}$, duration $10 \mathrm{~min}$. In the anodizing process, thin porous oxide layer was formed on the surface of $\mathrm{Al}$ foil. Then, the oxide layer was etched for $75 \mathrm{~min}$ in phosphoric-chromic acid solution $\left(35 \mathrm{ml} \mathrm{85 \%} \mathrm{H}_{3} \mathrm{PO}_{4}+\right.$ $\left.20 \mathrm{~g} \mathrm{CrO}_{3} / 1000 \mathrm{ml}\right)$ at $70^{\circ} \mathrm{C}$ to produce the nanostructured $\mathrm{Al}$ surface with regular pattern concaves and nanotips [16]. The array served as template for further processing.

The second anodizing process was performed in the same solution for the anodizing voltage $120 \mathrm{~V}$ to form desired porous alumina layer. This layer was etched further partially in the abovementioned acid solution to get the tips/ribs-like alumina morphology. In some cases, the anodizing/etching processing was repeated to get cone-like pores. The resulting samples were washed several times in deionized water and dried at $80{ }^{\circ} \mathrm{C}$.

\subsection{Deposition of hydrogenated silicon thin films}

Hydrogenated silicon (a/nc-Si:H) thin films were prepared using the plasma enhanced chemical vapor deposition (PE CVD) technique with the mixture of argon $(\mathrm{Ar})$, hydrogen $\left(\mathrm{H}_{2}\right)$ and silane $\left(\mathrm{SiH}_{4}\right)$ gases. The deposition parameters are shown in Table.

Parameters of Si:H thin film deposition.

\begin{tabular}{ll}
\hline Parameter & Value \\
\hline Microwave power & $700 \mathrm{~W}$ \\
Base pressure & $(1 \ldots 2) \cdot 10^{-5}$ Torr \\
Working pressure & $1.5 \cdot 10^{-2} \mathrm{Torr}$ \\
Substrate temperature & $200^{\circ} \mathrm{C}$ \\
Gas flow rates & \\
$\quad$ (a) Silane $\left(\mathrm{SiH}_{4}\right)$ & $100 \mathrm{sccm}$ \\
(b) Hydrogen & $1000 \mathrm{sccm}$ \\
(c) Argon $(\mathrm{Ar})$ & $1000 \mathrm{sccm}$ \\
Deposition time & $18 \mathrm{~min}$ \\
\hline
\end{tabular}

The hydrogen dilution ratio $\left[\mathrm{H}_{2}\right] /\left[\mathrm{SiH}_{4}\right]$ was kept constant at 10 , and the average deposition rate was about $10 \mathrm{~nm} / \mathrm{min}$.

\subsection{Characterization}

Raman spectra were measured with a Triplemate SPEX spectrometer equipped with a liquid nitrogen-cooled LN 1340PB (Princeton Instruments) multichannel CCD camera. The spectra were excited by a 488-nm argon laser line with a power at the sample surface of no higher than $5 \mathrm{~mW}$. The excitation wavelength was chosen so as to reduce the light penetration depth and prevent formation of the substrate spectrum, and the excitation power was low in order to preclude lightinduced sample crystallization. The sample was placed in the focal plane of a microscope which objective with an operation distance of $2 \mathrm{~mm}$ and an aperture of 0.6 , served to focus the laser beam and collect the scattered radiation. The laser beam spot on the sample surface was $2 \mu \mathrm{m}$ in diameter. In the experiments, $180^{\circ}$ scattering geometry was used. Raman spectra of films were measured with the resolution $5 \mathrm{~cm}^{-1}$. The photoluminescence spectra of the deposited films were measured using the Raman spectrometer. Nanocrystalline phase volume was determined from Raman spectra by using the method described in [17].

Morphologies of initial substrate and deposited layers were characterized using the scanning electron microscope (MIRA3 TESCAN).

\section{Results and discussion}

The properties of nanocrystalline silicon films are determined by surface morphology of the porous anodic alumina substrate. After the one-step anodization, PAA template has ripple-like surface morphology with a nanopore array. Typically, PAA layer has a duplex nature, which is composed of inner and outer sublayers [18]. In this case, PAA is very thin due to the short etching process. As a result, the pore walls are almost removed and only the inner oxide layer of about a few nanometers is observable. It is expected that the porous alumina layer is too weak and can be easily broken by mechanical stress such as bending.

In a two-step anodization, the self-organized nanopores array is created on the aluminum surface. The layer become thinner after partial etching, the inner and outer sublayers are retained in the pore structure of the porous anodic alumina substrate and aluminium surface acquires tipped/ribbed morphology. Thus, at anodization of aluminum foil by one-step and a two-step anodization, the surface and morphology of the porous anodic alumina, the structure of formed oxide is very different. A strong difference is also observed in the structure of silicon deposited on such a surface.

So, the films contain only amorphous phase, when they are deposited on ripple-like surface obtained with 
one-step anodization. Morphology of $\mathrm{Si}: \mathrm{H}$ films deposited on tipped/ribbed surface displays two separate phases. $\mathrm{Si}: \mathrm{H}$ films grown from the bottom of the oxide layer has smooth morphology, which is analogus to the $\mathrm{Si}: \mathrm{H}$ layer deposited at the one-step anodization. However, Si:H films deposited on the tipped region show columnar/wall structure, and this part consists of the nanocrystalline phase [18]. Therefore, it can be considered that there are two different growth types in the case of the films grown on tipped/ribbed surface, and the growth rate of nc-Si:H films is higher than that of a$\mathrm{Si}: \mathrm{H}$.

An important result of the studies of nanocrystalline silicon is the dependence of its structural properties on the pore size. Figs $1 \mathrm{a}$ and $1 \mathrm{~b}$ show the images of initial PAA templates after two-step anodization. As can be seen, the nanopores array of average diameter $452 \pm 35 \mathrm{~nm}$, interpore distance $500 \pm 24 \mathrm{~nm}$ and thickness of $1960 \pm 83 \mathrm{~nm}$ formed on the surface of aluminum. The phosphoric acid anodizing processing was chosen because of the following reasons. The alumina pores has their maximum size compared to other porous-forming electrolytes, and electrochemical processing in phosphoric acid could provide formation of duplex-layered oxide with anion species concentrated in the outer layer of the oxide. The species could form inclusions of wavellite $\mathrm{Al}_{3}(\mathrm{OH})_{3}\left(\mathrm{PO}_{4}\right)_{2} \cdot 5 \mathrm{H}_{2} \mathrm{O}$, augelite $\mathrm{Al}_{2}\left(\mathrm{PO}_{4}\right)(\mathrm{OH})_{3}$, or another aluminum phosphate hydroxides in alumina to facilitate its subsequent etching in phosphoric-chromic acid solution.
SEM image of silicon films with the thickness $200 \mathrm{~nm}$ deposited on an anodized porous aluminum substrate are shown in Fig. 2. In our case of deep pores, the a-S:H film is not deposited on the bottom of the pores. There is no amorphous phase at large depth of the pores, because the growth of amorphous phase starts at the bottom of shallow pores, and the nanocrystalline phase grows on the top of pores. In our case, the nanocrystalline nc-Si:H film grows above the top of the pores.

Raman spectroscopy demonstrates growing contribution of the crystalline phase with deposition duration, confirming the crystalline nature of the films (Fig. 3). The following Gaussian components were used for deconvolution of experimental Raman spectra. The first two ones, at 330 and $400 \mathrm{~cm}^{-1}$, represented the longitudinal acoustic (LA) phonon mode and longitudinal optical (LO) phonon of a-Si:H, correspondingly. The transversal optical (TO) phonon mode was deconvoluted into two bands: TO1 at $475 \mathrm{~cm}^{-1}$ for $\alpha-\mathrm{Si}: \mathrm{H}$, and TO2 at $490 \mathrm{~cm}^{-1}$ for intermediate nanocrystalline silicon phase. Crystalline silicon was represented by the rather sharp main peak $(\mathrm{CB} 1)$ at $521 \mathrm{~cm}^{-1}$. The crystallinity of the film $X_{c}=I_{c} / I_{a}$, where $I_{c}, I_{a}$ are the integrated intensities of the crystalline and amorphous peaks, was equal to $32 \%$.

The photoluminescence spectrum of the nanocrystalline silicon films demonstrated strong rather broad and pronounced peak at $\sim 2.21 \mathrm{eV}$ varying in height depending on the Si layer thickness (Fig. 4).
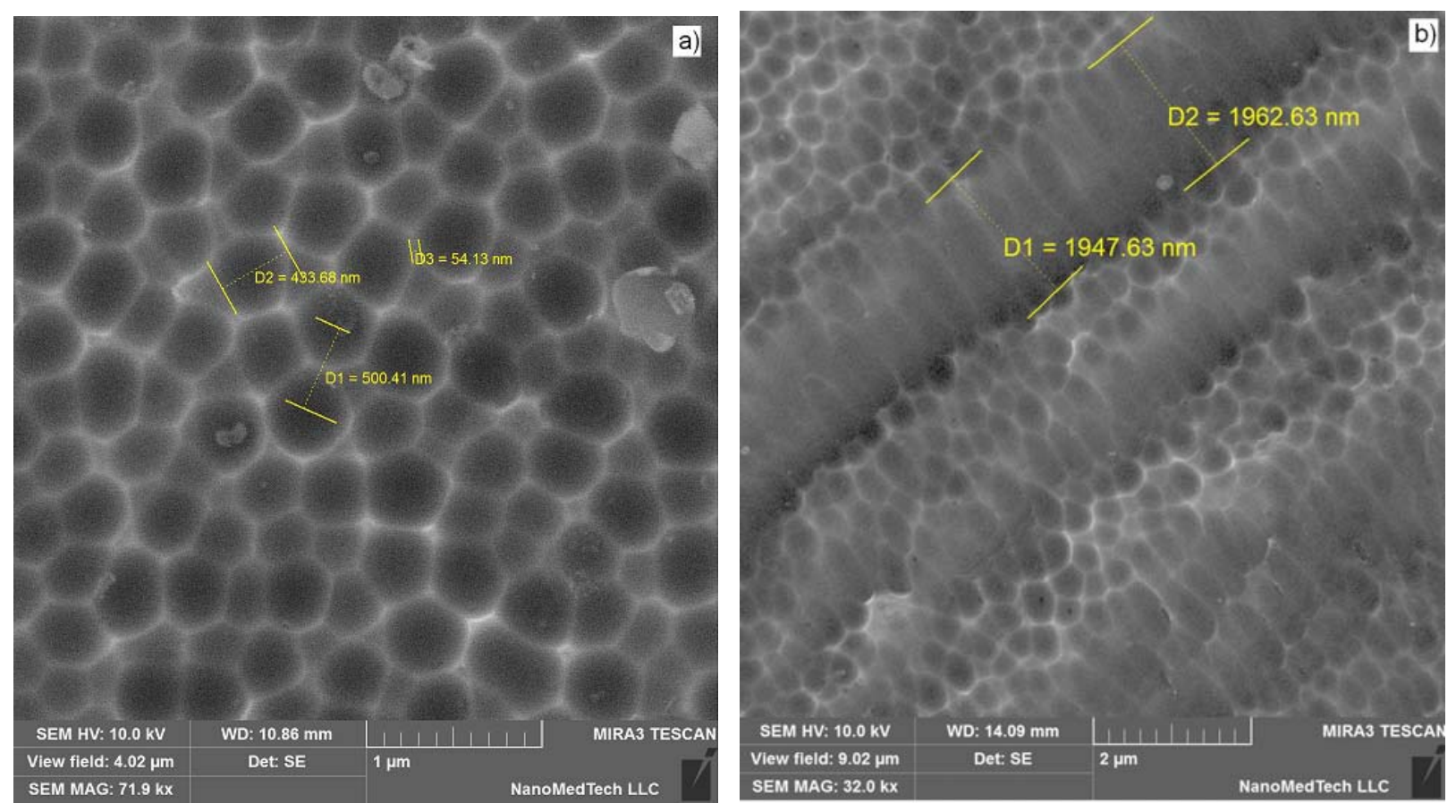

Fig. 1. SEM images of surface morphology inherent to initial PAA templates after two-step anodization: (a) plane view, (b) cross section view. 


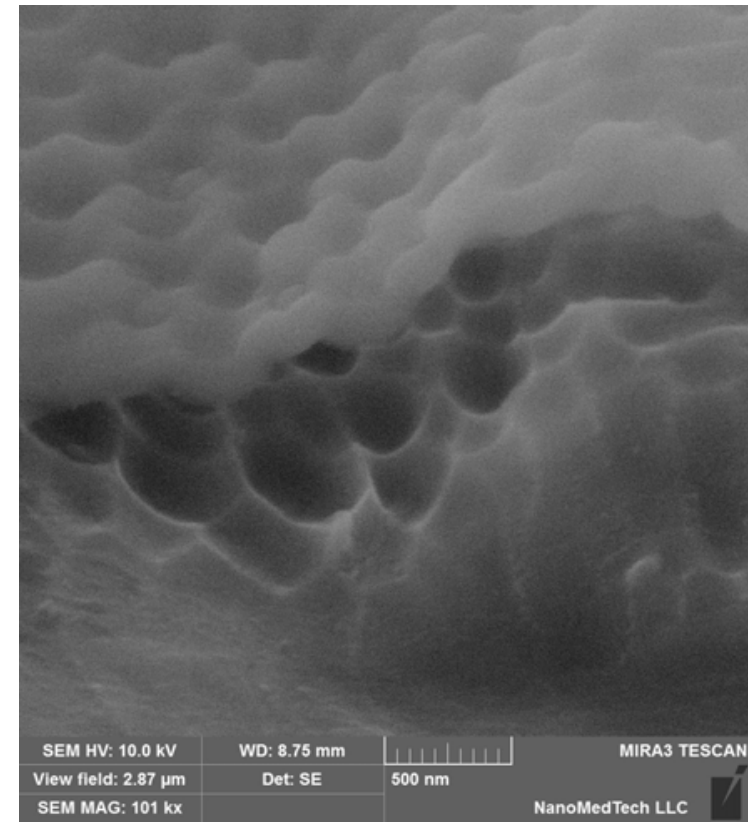

Fig. 2. SEM image of $\mathrm{Si}: \mathrm{H}$ thin film deposited on PAA templates after two-step anodization.

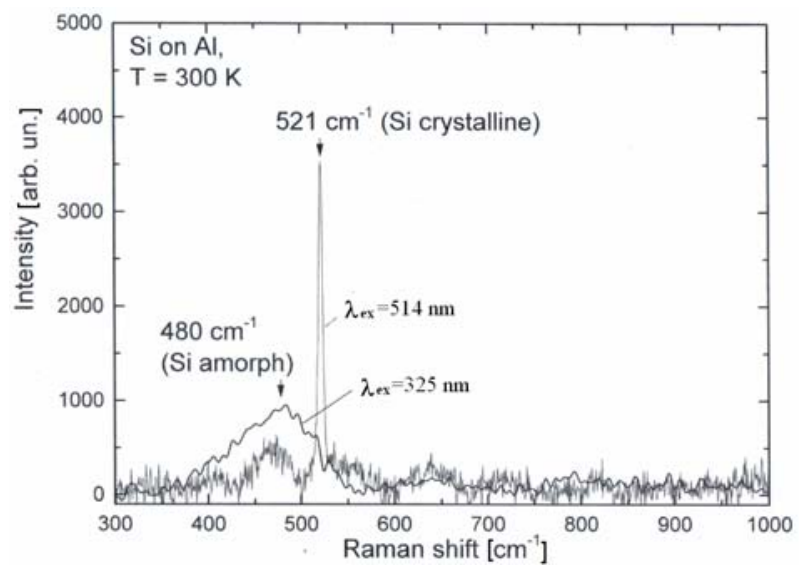

Fig. 3. Raman spectra of the nanocrystalline silicon films deposited on PAA templates ( $\lambda_{e x}$ is the wavelength of laser excitation).

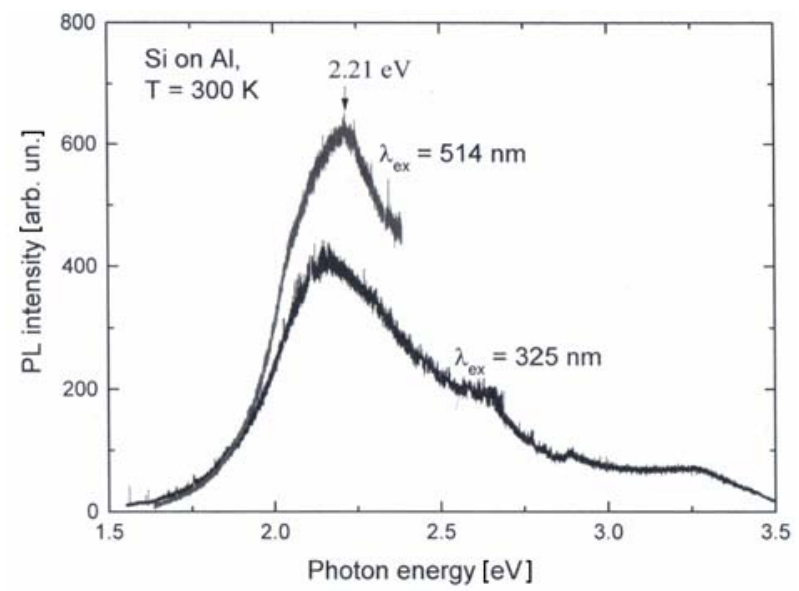

Fig. 4. PL spectra of the nanocrystalline silicon films deposited on PAA templates ( $\lambda_{e x}$ is the wavelength of laser excitation).
As it is known, correlation between Si nanocrystals diameter $d$ and photoluminescence peak energy $E_{\max }$ could be ascribed to quantum confinement and has the look [19]:

$$
E_{\max }=E_{g 0}+3.73[e V] /(d[n m])^{1.39},
$$

where $E_{g 0}$ is the band gap of bulk crystalline $\mathrm{Si}$. The size of nanocrystallites was estimated with account of the maximum position for the radiation peak and for $2.21 \mathrm{eV}$ was equal to $2.6 \mathrm{~nm}$.

\section{Conclusions}

The effect of the textured surface of PAA templates and pore size on the morphological and structural properties of hydrogenated silicon thin films grown using the PECVD method has been studied with SEM, Raman and photoluminescence spectroscopy. The $\mathrm{Si}: \mathrm{H}$ films deposited on ripple-like surface grows uniformly and consist mainly of amorphous phase. However, the film grown on the tipped/ribbed template shows novel morphology of heterogeneous a-Si:H/nc-Si:H mixed phase. The crystallinity of the layer and nanocrystallite sizes were weakly dependent on the pore size. A pure amorphous phase does not exist at a large depth of the pore.

\section{Acknowledgments}

This work was partially funded by State Fund for Fundamental Researches of Ukraine, grant F64/6-2016.

\section{References}

1. Hazar S., Ray S. Nanocrystalline silicon as intrinsic layer in thin film solar cells. Solid State Communs. 1998. 109. P. 125-128.

2. Schropp R.E.I., Rath J.K., Li H. Growth mechanism of nanocrystalline silicon at the phase transition and its application in thin film solar cells. J. Crystal Growth. 2009. 311. P. 760-764.

3. S. Guha, J. Yang, B. Yan, High efficiency multijunction thin film silicon cells incorporating nanocrystalline silicon. Solar Energy Materials \& Solar Cells. 2013. 119. P. 1-11.

4. Nasuno J., Kondo M., Matsuda A., Key M. Issue for the fabrication of high-efficiency microcrystalline silicon thin-film solar cells at low temperature. Jpn. J. Appl. Phys. 2002. 41. P. 5912 5915.

5. Shah A., Meier J., Vallat-Sauvain E., Droz C., Graf U. Microcrystalline silicon and micromorph tandem solar cell. Thin Solid Films. 2002. 179. P. 403-404.

6. Hsiao H.L., Hwang H.L., Yew T.R. Study on low temperature faceting growth of polycrystalline silicon thin film by ECR downstream plasma CVD 
with different hydrogen dilution. Appl. Surf. Sci. 1999. 142. P. 316-322.

7. Scott B.A., Reimer J.E., Reuter W. Low defect density amorphous hydrogenated silicon prepared byhomogeneous chemical vapor deposition. Appl. Phys. Lett. 1982. 40. P. 973.

8. Acciarri M., Binetti S., Bollani M., von Kane H. Nanocrystalline silicon film growth by LE-PECVD for photovoltaic application. Sol. Energ. Mat. Sol. C. 2005. 87. P. 11-16.

9. Fonrodona M., Soler D., Escarre J., MohammedBrahim T. Low temperature amorphous and nanocrystalline silicon thin film transistors deposited by Hot-Wire CVD on glass substrate. Thin Solid Films. 2006. 501. P. 303-308.

10. Saleh R., Nickel N.H. Raman spectroscopy of Bdoped microcrystalline silicon films. Thin Solid Films. 2003. 427. P. 266.

11. Das D. A novel approach towards silicon nanotechnology. J. Phys. D: Appl. Phys. 2003. 36. P. 2335-2340.

12. Pevtsov A.B., Feoktistov N.A. Photoluminescence of thin amorphous-nanocrystalline silicon film. Tech. Phys. Lett. 2002. 28/4. P. 305-311.
13. T Shimizu., Nakazawa K., Kumeda M., Veda S. Defects in hydrogenated amorphous silicon films prepared by glow discharge decomposition and sputtering. Physica B/C. 1983. 117/118. P. 926.

14. Santos P., Johnson N., Stree R. Light-enhanced hydrogen motion in a-Si:H. Phys. Rev. Lett. 1991. 67. P. 2686-2691.

15. Khodin A., Lee J.K., Kim C.S. Nanomorph silicon grown on template alumina substrate by plasmaenhanced CVD. Mat. Lett. 2009. 63. P. 2552-2555.

16. Kizjak A.Yu., Evtukh A.A., Steblova O.V. Formation of nanoporous $\mathrm{Al}_{2} \mathrm{O}_{3}$ films. Sensor Electron. and Microsyst. Technol. 2016. 13. P. 83-92.

17. Gaisler S.V., Semenova O.I., Sharafutdinov R.G., Kolesov B.A. Analysis of Raman spectra of amorphous-nanocrystalline silicon films. Phys. Solid State. 2004. 46, No. 8. P. 1528-1532.

18. Khodin A., Lee J.K., Kim C.S. Hydrogenated amorphous/nanocrystalline silicon thin films on porous anodic alumina substrate. Sur. Rev. and Lett. 2010. 17. P. 283-287.

19. Voigt F., Briggermann R., Unold T., Huisken F. Porous thin films grown from size-selected silicon nanocrystals. Mater. Sci. Eng. C. 2006. 25. P. 584 589. 